\title{
Ghost AND Noise Removal in Exposure Fusion For High DyNamic RANGE IMAgING
}

\author{
Dong-Kyu Lee ${ }^{1}$, Rae-Hong Park ${ }^{1}$ and Soonkeun Chang ${ }^{2}$ \\ ${ }^{1}$ Department of Electronic Engineering, School of Engineering, Sogang University, \\ 35 Baekbeom-ro (Sinsu-dong), Mapo-gu, Seoul 121-742, Korea \\ ${ }^{2}$ Samsung Electronics Co., Ltd., Suwon, Gyeonggi-do 443-742, Korea
}

\begin{abstract}
For producing a single high dynamic range image (HDRI), multiple low dynamic range images (LDRIs) are captured with different exposures and combined. In high dynamic range (HDR) imaging, local motion of objects and noise in a set of LDRIs can influence a final HDRI: local motion of objects causes the ghost artifact and LDRIs, especially captured with under-exposure, make the final HDRI noisy. In this paper, we propose a ghost and noise removal method for HDRI using exposure fusion with subband architecture, in which Haar wavelet filter is used. The proposed method blends weight map of exposure fusion in the subband pyramid, where the weight map is produced for ghost artifact removal as well as exposure fusion. Then, the noise is removed using multi-resolution bilateral filtering. After removing the ghost artifact and noise in subband images, details of the images are enhanced using a gain control map. Experimental results with various sets of LDRIs show that the proposed method effectively removes the ghost artifact and noise, enhancing the contrast in a final HDRI.
\end{abstract}

\section{KEYWORDS}

High Dynamic Range Imaging, Exposure Fusion, Ghost Removal, Noise Removal

\section{INTRODUCTION}

Digital sensors and display devices such as digital camera, television, etc., have a limited dynamic range. They cannot capture and display the full dynamic range with which people can perceive a real world scene. For example, when a scene in which bright and dark regions coexist is captured, these regions tend to be under- or over-saturated because of the limited dynamic range. The dynamic range is one of the important criteria for evaluating image quality, especially in devices supporting high resolution images. Images and display devices supporting high dynamic range (HDR) are attractive to producers and customers of today.

In order to acquire a HDR image (HDRI), HDR imaging techniques were proposed [1-5]. Multiple low dynamic range images (LDRIs) are captured with multiple exposures using auto exposure bracketing of a camera. Then, captured LDRIs are combined into a single HDRI by HDR imaging. However, when LDRIs are combined, several problems can occur due to global and local motions [6-9] of a camera or an object, and noise [10-12] in LDRIs. The ghost artifact and noise are major problems in HDR imaging. Since HDRI is generated from multiple images, moving camera or object causes the ghost artifact. HDR imaging is generally used for highquality image even in the low-light or back-light condition in which captured images tend to have much noise due to camera setting with short-exposure time or high sensitivity. Therefore, the noise is also one of the critical issues in HDR imaging.

DOI : 10.5121/ijcga.2014.4401 
HDR imaging can be generally classified into two approaches. In the first approach, HDR imaging [1-3] consists of radiance map generation and tone-mapping. First, a HDR radiance map, which covers the entire dynamic range of LDRIs, is generated [1]. Generally, in this radiance map generation process, the ghost artifact due to local motion and noise are removed [2, 3, 11]. Then, the radiance-map is tone-mapped back to a LDR representation to fit the range of display or printing devices [13]. The second approach is image fusion, where LDRIs are blended directly into a single HDRI using weight map [4, 5, 14-17]. To remove the ghost artifact due to local motion and noise, the weight map is computed using image quality measures such as ghost and noise as well as contrast, well-exposedness, and saturation.

In this paper, we propose a ghost and noise removal method using exposure fusion for HDR imaging. In the proposed method, exposure fusion is used in the subband architecture, where exposure fusion blends directly LDRIs using the weight map guided by quality measures for HDR effect. To generate motion maps for removing ghost artifact, the proposed histogram based motion maps [18] are used, where the motion maps are combined with the weight maps of exposure fusion. Fused subband images are denoised by multi-resolution bilateral filtering [19], which is very effective in removing noise. After denoising, details of the subband images are enhanced through the gain control [20]. Next, detail-preserved subband images are reconstructed to a single final fused image.

The rest of the paper is organized as follows. In Section 2, image fusion for HDR imaging, ghost removal methods, and noise reduction methods in HDR imaging are reviewed. Section 3 proposes an exposure fusion method using subband architecture. In this section, the proposed histogram based ghost removal method, noise reduction method, and gain control method in subband architecture are described. Experimental results of the proposed and existing ghost and noise removal methods for HDR imaging are compared and discussed in Section 4. Finally, Section 5 concludes the paper.

\section{Previous Work}

To get a single HDRI, LDRIs are captured by exposure bracketing in a camera and combined in HDR imaging [1-5]. However, image quality is rather degraded in HDR imaging unless some artifacts such as ghost artifact and noise are reduced. In this section, we review previous work on HDR imaging, ghost removal, and noise reduction.

\subsection{Image Fusion for HDR Imaging}

Image fusion for HDR imaging skips the process of generating an HDR radiance map, and directly fuses a set of multi-exposed images to a single HDRI [4]. It measures the quality of each pixel in LDRIs and computes weighted average guided by quality measures for high-quality image. It has several advantages that it is implemented in a simple acquisition pipeline and does not require to know exposure times of every LDRI and to calculate the camera response curve with the exposure times. Compared with the case in which a single image is used for HDR imaging [21], image fusion enhances better contrast and dynamic range because more information such as contrast, detail, and structure in the images can be used.

Goshtasby [4] proposed a block-based fusion method for HDR imaging. This method selects blocks that contain the most information within that block and then the selected blocks are blended together using blending function that is defined by rational Gaussian. Raman and 
Chaudhuri [15] proposed a bilateral filter based composition method. They computed the difference between LDRIs and the bilateral filtered image, and designed weight function using the difference, where weak edges and textures are given high weight values to preserve detail. $\mathrm{Li}$ et al. [22] proposed a layered-based fusion algorithm. They used a global-layer to improve the robustness and color consistency, and the gradient domain was used to preserve details. Bertalmio and Levine [23] introduced an energy function to preserve edge and color. They measured the difference in edge information in the short-exposure image while the local color difference in the long-exposure image.

In exposure fusion [5], quality measures for contrast, saturation, and well-exposedness are used to compute the weight maps for each LDRI. Then, the weight map and LDRIs are fused using Gaussian and Laplacian pyramid decomposition, respectively. Shen et al. [20] presented a detailpreserving exposure fusion. They applied the exposure fusion [5] to subband architecture using quadrature mirror filter (QMF), in which the gain control strategy [24] was used to preserve details in HDRI.

\subsection{Ghost Artifact Removal}

Ghost artifact is due to global motion of a camera and local motion of objects in a scene. Even though image registration $[6,25,26]$ is used to remove global motion with a hand-held camera, ghost artifact still remains due to local motion of objects, because LDRIs are not captured simultaneously and moving objects can be located at different positions during taking LDRIs. Figure 1 illustrates an example of the ghost artifact in HDRI. Figure 1(a) shows three LDRIs (Bench, 1168 $\times 776$ ) with different exposures after image registration [11]. Note that illuminations of images and object positions are changed according to exposure time of the LDRIs. Figure 1(b) shows the final HDRI using exposure fusion [5]. Although the dynamic range in the image is extended, the ghost artifact appears in region that contains a moving object.
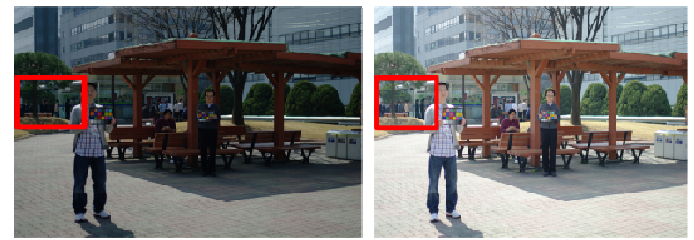

(a)

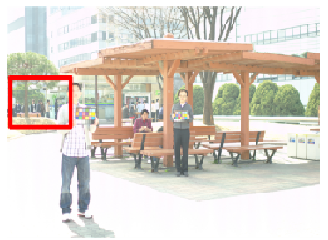

1

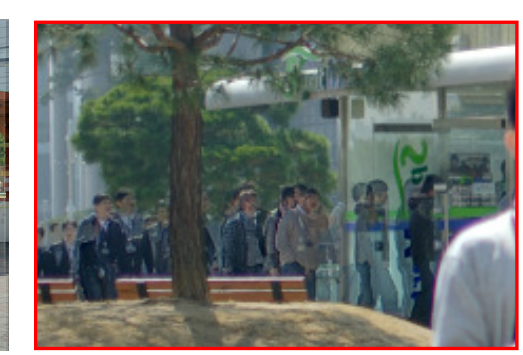

(b)

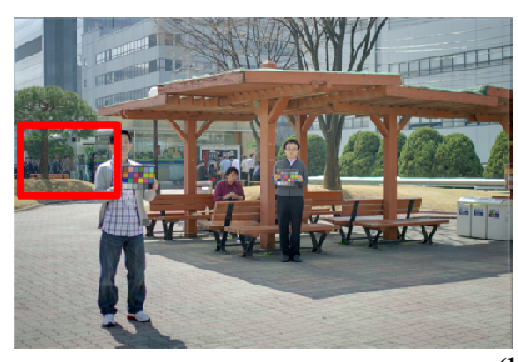

Figure 1. Example of the ghost effect in HDRI imaging. (a) three LDRIs $(1168 \times 776$, Bench), (b) HDRI using exposure fusion [5] and its cropped image. 
International Journal of Computer Graphics \& Animation (IJCGA) Vol.4, No.4, October 2014

To generate the motion map, region with large intensity change can be simply considered as region of object motion. However, LDRIs are captured with different exposure times, thus this method is not directly applicable to HDR imaging and the illumination change between LDRIs should be considered. Therefore, the removal method of the ghost artifact caused by local motion uses the property, which is not sensitive to exposure time, such as variance, entropy, and histogram between LDRIs.

To detect object motion, a variance based method [2] uses the weighted variance of pixel values between multiple-exposure LDRIs and divides images by thresholding into two regions: motion region and background. On the other hand, Jacobs et al. [3] used the difference of local entropy between multiple-exposure LDRIs to detect motion region. The measure using the difference of local entropy is effective for detecting the features such as intensity edges and corners regardless of exposures. However, it is sensitive to parameter values used to define motion and to the window size for the local entropy. Khan et al. [27] proposed the ghost artifact removal method based on the kernel density estimator. They computed the probability that the pixel is contained in the background and used the probability as a weighting factor for constructing the radiance map.

Jia and Tang [28] used a voting method for color/intensity correction of input images. In this method, global and local replacement functions are iteratively estimated using intensity values in voting space and the replacement functions are employed to detect occlusion which causes ghost artifact. While this method gives a high contrast image from defective input images with global and local alignment, the computational load is high because of optimization process for computing the replacement function.

Histogram based methods $[7,11]$ classify the intensity values into multi-levels. They regard the region with large difference between the level indices as motion region. The computational load of the methods is relatively low, however they excessively detect wrong region by dividing the intensity range into a number of levels.

\subsection{Noise Removal}

Tico et al. [14] proposed a noise and blur reduction method in HDR imaging. They used the property of LDRIs that LDRIs captured with under-exposure are noisy, whereas those with overexposure are blurred. They first photometrically calibrated LDRIs using brightness transfer function between the longest exposure image and the remaining shorter exposure images, and fused calibrated LDRIs with noise estimation in the wavelet domain. In the fusion step, the weighted average is used, where the larger noise variance of the pixel neighborhood is, the smaller the computed weight of the pixel is.

Akyuz and Reinhard [10] reduced noise in radiance map generation of HDR imaging, in which input LDRIs captured at high sensitivity setting were used. They first generated the radiance map of each LDRI using an inverse camera response curve, and computed the pixel-wise weighted average of subsequent exposure images to reduce the noise. The weighting function depends on exposure time and pixel values. They gave more weight to pixels of LDRIs captured with longer exposure, but excluded over-saturated pixels from the averaging.

Min et al.'s method [11] selectively applies different types of denoising filters to motion regions and static regions in radiance map generation that is based on Debevec and Malik's method [1]. In motion regions, a structure-adaptive spatio-temporal smoothing filter is used, whereas in static regions, a structure-adaptive spatial smoothing filter is used for each LDRI and then the weighted averaging for filtered LDRIs is performed. This filter is effective for low-light noise removal with edge preservation and comparably low computational load 


\section{HDRI GENERATION}

The proposed method is based on the subband architecture [20]. Exposure fusion [5] with ghost removal [18] for HDR, denoising [19], and gain control [24] to enhance detail are performed in the subband framework of the proposed method. Figure 2 shows a block diagram of the proposed HDR imaging framework using subband architecture [20], where thick lines represent more than two images or subband images, whereas thin lines represent a single image or subband image. Given $k$ th exposure LDRIs $I_{k}(1 \leq k \leq K)$, they are decomposed into a number of subband images $X_{k}^{i}(1 \leq i \leq 3 L+1)$ by analysis filter. With decomposed subband images, exposure fusion is performed, where the motion maps $M_{k}^{\prime}$ are generated and then combined with the weight maps of exposure fusion for ghost artifact removal. Fused subband images $F^{i}$ are denoised by multiresolution bilateral filter and soft thresholding, where the lowest-frequency subband image $F^{3 L+1}$ and the high-frequency subband images $F^{j}(1 \leq j \leq 3 L)$ are denoised by bilateral filtering and soft thresholding, respectively. Denoised subband images are denoted as $\hat{F}^{i}$. Next, the gain control map for detail preserving is computed and applied to denoised subband images. Finally, detail preserved subband images $\widetilde{F}^{i}$ are reconstructed to a single final result image $I^{\prime}$ by synthesis filer.

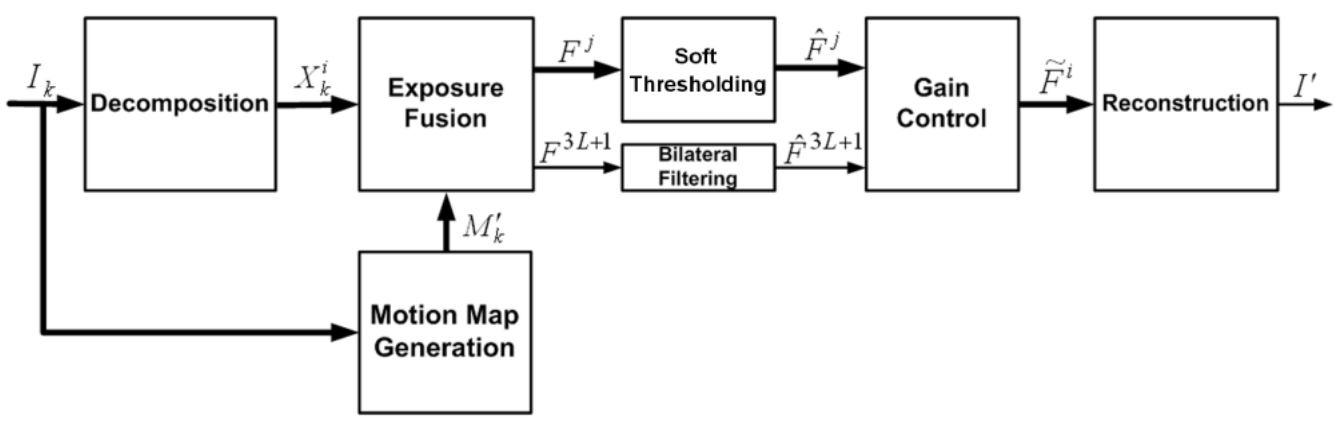

Figure 2. Block diagram of the proposed HDR imaging framework using subband architecture.

This section is organized as follows. In Section 3.1, exposure fusion using subband architecture is described. Sections 3.2 and 3.3 present the proposed histogram based ghost removal method and denoising method using multi-resolution bilateral filter, respectively. In Section 3.4, to preserve and enhance detail of a final image, gain control method is described.

\subsection{Exposure Fusion Using Subband Architecture}

The proposed method is based on exposure fusion [5], in which pixel-wise quality measures for contrast, saturation, and well-exposedness are defined. Contrast measure $C$ is computed as the absolute value of the Laplacian filter response to the grayscale of each image. The use of this measure makes edges and details preserved. Saturation measure $S$ is defined as the standard deviation within the color channel to assign a higher weight to pixels with more saturated color. Well-exposedness measure $E$ uses a Gaussian curve, where the closer the pixel value is to 0.5 , the higher the weight is given, where the pixel value is normalized to the range 0 to 1 . Gaussian curve is applied to each color channel, and results of three (red, green, and blue) color channels are multiplied. Three measures at pixel $(x, y)$ in $k$ th exposure image are combined using multiplication to produce the weight map 


$$
W_{k}(x, y)=\left(C_{k}(x, y)\right)^{w_{C}} \times\left(S_{k}(x, y)\right)^{w_{S}} \times\left(E_{k}(x, y)\right)^{w_{E}}
$$

where the subscript $k$ represents $k$ th exposure image and the influence of three measures of contrast, structure, and well-exposedness are controlled by power terms $w_{C}, w_{S}$, and $w_{E}$, respectively. For consistent result, the weight map is normalized as

$$
\hat{W}_{k}(x, y)=\frac{W_{k}(x, y)}{\sum_{k=1}^{K} W_{k}(x, y)}
$$

where $K$ is the total number of LDRIs used to fuse for generating a single HDRI.

In exposure fusion [5], LDRIs and the weight maps are separately fused using a multi-resolution

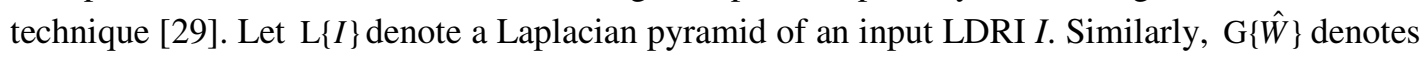
a Gaussian pyramid of the normalized weight map $\hat{W}$. The Laplacian pyramid of the final fused HDRI is generated as

$$
\mathrm{L}\left\{I^{\prime}\right\}^{l}=\sum_{k=1}^{K} \mathrm{G}\{\hat{W}\}_{k}^{l} \mathrm{~L}\{I\}_{k}^{l}
$$

where the superscript $l(1 \leq l \leq L)$ represents the layer index in a pyramid. A final HDRI $I^{\prime}$ is

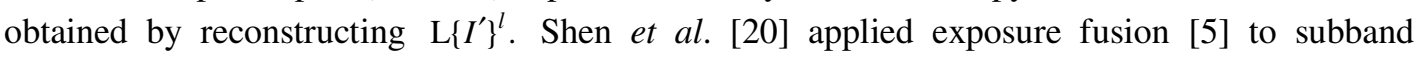
architecture using QMF. They built the subband pyramid through an $L$-level QMF for decomposing LDRIs and blended the subband images with the weighted maps of exposure fusion [5]. The blended subband images are modified according to gain control maps [24] to preserve details of the subband signals. A fused HDRI is reconstructed using detail-preserved subband images.

The proposed method constructs the subband architecture with Haar wavelet filter and blends weight maps of exposure fusion in the subband pyramid. Next, the proposed method computes the gain control map to control the strength of the subband signals and modifies all the subbands using the gain control map. The modified subband images are reconstructed using the subband pyramid to a final result image.

For $L$-level subband pyramid, subband images $X_{k}^{i}(x, y)$ of $k$ th exposure LDRI $I_{k}$ are generated. To obtain a fused subband image $F^{i}$, the proposed method computes a weighted average of $X_{k}^{i}$ at each pixel of subband image as

$$
F^{i}(x, y)=\sum_{k=1}^{K} \tilde{W}_{k}(x, y) X_{k}^{i}(x, y)
$$

where $\tilde{W}_{k}$ is a Gaussian filtered version of the normalized weight map $\hat{W}_{k}$, which prevents undesirable halo around edge. This weighted averaging function makes a fused image have good image quality defined by the quality measures. Each subband image contains image features through image filtering. Thus, the weighted averaging blends image features instead of intensity using the subband architecture, which is effective for avoiding seams. 
International Journal of Computer Graphics \& Animation (IJCGA) Vol.4, No.4, October 2014

\subsection{Histogram Based Ghost Removal}

In the proposed method for motion map generation [18], the histogram based multi-level threshold map [7] is extended to remove the ghost artifact caused by local motion. When contrast between moving objects and background is small, or the textureless objects move, these motions give relatively large intensity change than that of variance and local entropy [7]. Thus, the intensity histogram provides useful methods for local motion detection. The multi-level threshold map was extended from a median threshold bitmap (MTB), which is a binary bitmap constructed by partitioning an image using median pixel value of the image as a threshold [6]. The median threshold is robust to illumination change and thus useful for comparing LDRIs. With this robustness, the multi-level threshold map [7] was proposed to detect local motion more accurately than MTB. This method classifies the intensity values into multi-levels and then finds motion region using the difference between the level indices of intensity values, whereas MTB classifies pixels into two levels according to intensity value.

The multi-level threshold method [7] detects better local motion than MTB, however, this method may excessively detect wrong region by dividing the intensity range into more than two levels. Since its excessive detection finds not only motion region but also region without motion as motion region, the effect on HDR imaging can be reduced. To avoid this drawback, the method that adaptively detects motion was introduced [11]. The method separates the LDRI into three regions: no difference region, small difference region, and large difference region. In the small difference region, pixels that are connected to the large difference region are selected as motion region of the large difference. However, the adaptive motion selection has a problem of how to divide the LDRI into the three regions, because it is difficult to decide which pixels in small difference region are selected as motion region.

To detect local motion, the proposed motion map [18] is generated based on rank according to the intensity. It is assumed that the ranks of intensity within an LDRI are similar to those of the other LDRIs except for the local motion region. Let $r_{k}(x, y) \quad\left(1 \leq r_{k} \leq R_{k}\right)$ be rank at pixel $(x, y)$ of $k$ th exposure image. It can be expressed using a cumulative distribution function of intensity value at pixel $(x, y)$. The rank $r_{k}(x, y)$ is normalized to $N$ bit as

$$
\hat{r}_{k}(x, y)=\text { floor }\left(\frac{r_{k}(x, y)}{R_{k}} \times 2^{N}\right), 1 \leq r_{k} \leq R_{k}
$$

where $R_{k}$ is the last rank in $k$ th exposure image and floor $(x)$ represents the floor function that maps a real number to the largest integer smaller than or equal to $x$. $N$ is selected considering hardware costs, where larger $N$ gives better performance in a final HDRI, but requires higher computational cost. It is assumed that the difference of normalized ranks between intensity values at pixel $(x, y)$ in the reference LDRI and $k$ th exposure image is due to local motion. A reference image serves as reference for detection of non-static regions. The reference image can be selected as an image in which area of non-saturated region is the largest among LDRIs, or be produced using pre-processing such as photometric calibration. However, we assume that in general the middle-exposure LDRI is well-exposed and saturated region of the image is the least, thus we select the middle-exposure LDRI as the reference image.

Then, the absolute difference of normalized ranks is computed as

$$
d_{k}(x, y)=\left|\hat{r}_{r e f}(x, y)-\hat{r}_{k}(x, y)\right|
$$


where the subscript ref represents the middle-exposure. Using $d_{k}$, the motion maps $M_{k}$ are defined as

$$
M_{k}(x, y)= \begin{cases}0, & \text { for } d_{k}(x, y) \geq T_{\text {rank }}, k \neq r e f \\ 1, & \text { otherwise }\end{cases}
$$

where $T_{\text {rank }}$ is the threshold value selected to reduce error caused by normalized rank change between LDRIs due to different exposures or non-ideal intensity change. In the motion maps, the pixel with zero (0) value represents the pixel with motion.

The motion maps are clustered by applying the morphological operations [30] which are needed to avoid partial detection of a moving object. First, isolated pixels are removed, and then disconnected pixels are linked. Finally, the holes that are surrounded by 1-value pixels are filled. After these morphological operations, the final motion maps are denoted as $M_{k}^{\prime}(x, y)$. The final rank based motion maps $M_{k}^{\prime}(x, y)$ are combined into the weight map in exposure fusion using multiplication as

$$
W_{k}^{\prime}(x, y)=\left(C_{k}(x, y)\right)^{w_{C}} \times\left(S_{k}(x, y)\right)^{w_{s}} \times\left(E_{k}(x, y)\right)^{w_{E}} \times M_{k}^{\prime}(x, y) .
$$

This weight map $W_{k}^{\prime}(x, y)$ is normalized and filtered to produce $\tilde{W}_{k}(x, y)$ in (4). Using the weighted average of $X_{k}^{i}$ by $\tilde{W}_{k}$, the proposed method generates ghost removed subband images $F^{i}(x, y)$.

\subsection{Noise Removal Using Multi-Resolution Bilateral Filtering}

The proposed method removes noise in subband architecture. The subband images decomposed by the wavelet filter are effectively used for noise removal. In wavelet domain, whereas Tico et al. [14] only estimated noise variance at every pixel in all LDRIs using the robust median estimator [31] and then computed weights of each pixel according to the noise variance, the proposed method estimates and removes the noise in the subband images of a single fused subband image $F^{l}$ using Zhang and Gunturk's method [19].

Zhang and Gunturk's method [19] combines multi-resolution bilateral filtering with wavelet thresholding for image denoising. This method decomposes an image into low- and highfrequency subbands as the proposed method. The bilateral filter [32] is an edge-preserving denoising filter, where the intensity value at each pixel is replaced by Gaussian weighted average of intensity values of nearby pixels. This filter is applied to the lowest-frequency subband image. Wavelet thresholding [33] can remove noise components with hard or soft thresholding operations. Soft thresholding is applied to the high-frequency subband image. Bilateral filter and wavelet thresholding are also used to provide an effective noise reduction method with visibleband and wide-band image pair in Yoo et al.'s method [34].

The proposed method applies the bilateral filter to the lowest-frequency subband image $F^{3 L+1}$ and then obtains bilateral filtered image $\hat{F}^{3 L+1}$. On the other hand, the high-frequency subband images $F^{j}(1 \leq j \leq 3 L)$ are filtered by soft thresholding 
International Journal of Computer Graphics \& Animation (IJCGA) Vol.4, No.4, October 2014

$$
\hat{F}^{j}(x, y)= \begin{cases}0, & \text { if }\left|F^{j}(x, y)\right|<T_{\text {soft }} \\ \left.\operatorname{sgn}\left(F^{j}(x, y)\right)|| F^{j}(x, y) \mid-T_{\text {soft }}\right), & \text { otherwise }\end{cases}
$$

where $T_{\text {soft }}$ is a threshold in soft thresholding. Soft thresholding suppresses the noise by applying nonlinear transform to the wavelet coefficients.

\subsection{Gain Control Map}

After obtaining the fused subband images, the proposed method uses an approach similar to Li et al.'s method [24] and Shen et al.'s method [20] to compute the gain control map. Generally, a defect of a denoised image is that detail and texture parts in image are degraded during denoising. Thus, strong parameter is not used in many denoising algorithms, in which strong denoising filter is designed though. Therefore, detail enhancement is essential for restoring the artifacts by denoising. The proposed method preserves the detail and texture parts in subband architecture without converting other architectures. All processing, i.e., exposure fusion, ghost removal, noise reduction and detail preservation are performed in a single architecture, where they are closely related to each other.

When the photoreceptors adapt to the new light level, this process can be modeled as the logarithm of the input intensity. Thus, the proposed method controls subband signals using the logarithm of the input intensity, where the high contrast in areas with abruptly changing illumination is reduced and the details in texture regions are preserved.

To compute the gain control map, an activity map $A^{i}(1 \leq i \leq 3 L+1)$ [24] of $i$ th subband image from absolute values of local filter response is constructed as

$$
A^{i}(x, y)=g(x, y, \sigma) *\left|\hat{F}^{i}(x, y)\right|
$$

where Gaussian filter $g(x, y, \sigma)$ with subband dependent scale parameter $\sigma$ is used. A gain control map $G$ is defined using a gamma-like function from the activity map as

$$
G^{i}(x, y)=\left(\frac{A^{i}(x, y)+\varepsilon}{\delta^{i}}\right)^{(\gamma-1)}
$$

where $\gamma$ is a weighting factor between 0 and 1 , and $\varepsilon$ prevents singularity in the gain map. This mapping is a monotonically decreasing function, where if the activity is high, gain is decreased, otherwise, increased. $\delta$ is used as a gain control stability [24].

The gain control maps are aggregated to a single gain control map [24], because each activity in the subband is correlated with those in adjacent subbands. Thus, an activity map $A^{a g}(x, y)$ aggregated over scales and orientations is computed as

$$
A^{a g}(x, y)=\sum_{i=1}^{3 L+1} A^{i}(x, y)
$$

and from which, a single gain control map is derived as 
International Journal of Computer Graphics \& Animation (IJCGA) Vol.4, No.4, October 2014

$$
\begin{gathered}
G^{a g}(x, y)=\left(\frac{A^{a g}(x, y)+\varepsilon}{\delta}\right)^{(\gamma-1)}, \\
\delta=\alpha \frac{\sum_{(x, y)} A^{a g}(x, y)}{\text { Width } \times \text { Height }}
\end{gathered}
$$

where Width and Height are width and height of $\hat{F}$, respectively. The single gain control map $A^{a g}(x, y)$ is applied to fused subband images $\hat{F}^{l}(x, y)$ to get $\tilde{F}^{i}(x, y)$

$$
\tilde{F}^{i}(x, y)=m^{i} G^{a g}(x, y) \times \hat{F}^{i}(x, y)
$$

where $m^{i}$ controls the modification extent of different frequency according to scale of the subband [24]. Finally, the gain controlled subband images $\tilde{F}^{i}$ are reconstructed to a single final result image $I^{\prime}$ by a wavelet synthesis filer.

\section{EXPERIMENTAL RESUltS AND DISCUSSIONS}

We have tested the proposed method using various sets of real LDRIs with different exposures. Before simulating the proposed method and other ghost and noise removal methods for HDR, input images are aligned by a registration method used in [11] to reduce the distortion caused by global motion.

We compare the performance of the proposed and existing ghost and noise removal methods in views of subjective quality for HDR imaging, ghost artifact removal, noise removal, and computation time.

\subsection{HDR Imaging}

Figure 3 show comparison of HDR results with and without gain control (House, $752 \times 500$ ). Figure 3(a) shows input LDRIs with three different exposures. Figures 3(b) and 3(c) show results by the proposed method without and with gain control, respectively, where the effect of HDR imaging from three input LDRIs is produced. Edge and details in result image by the proposed method with gain control in Figure 3(c) are stronger than those shown in Figure 3(b). By the gain control, the proposed method can give detail-preserving results for a HDRI.
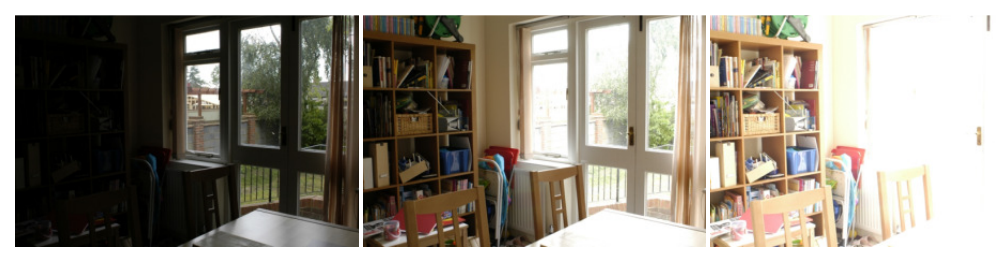

(a) 


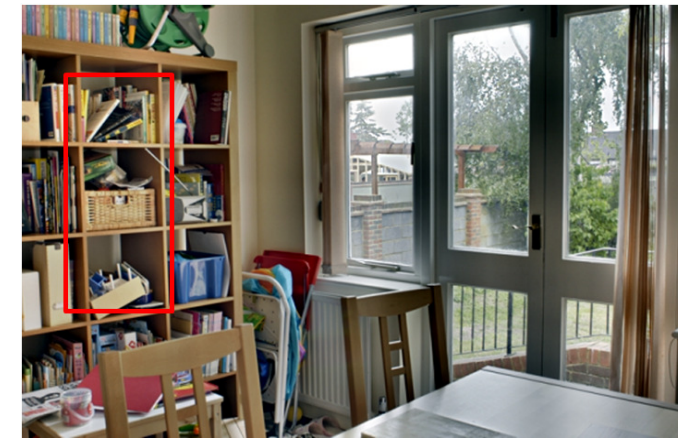

(b)

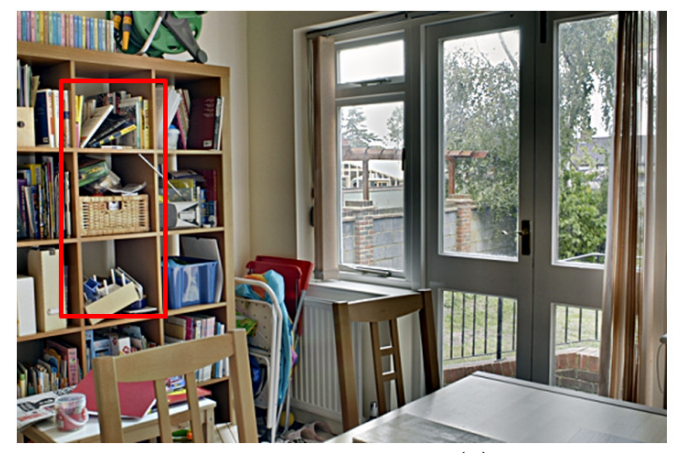

(c)
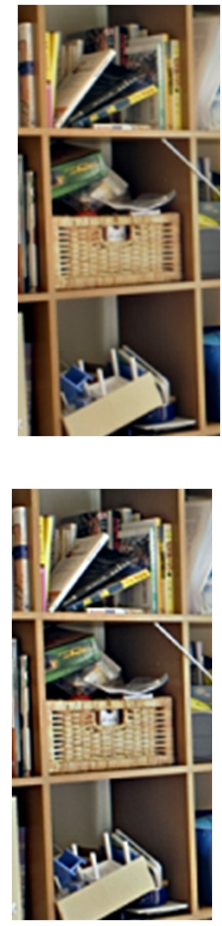

Figure 3. Comparison of HDR results with and without gain control $(752 \times 500$, House). (a) input LDRIs, (b) result by the proposed method without gain control, (c) result by the proposed method with gain control.

In Figure 4, the performances of the ghost removal methods are compared in view of HDR imaging. Because the ghost removal methods may excessively detect wrong regions, the performances of the ghost removal methods are evaluated by comparing area of HDR imaging region. Three input LDRIs (Field, $728 \times 546$ ) are used. Figures $4(\mathrm{a})-4(\mathrm{~d})$ show results of variance based method [2], Jacobs et al.'s method [3], adaptive multi-level threshold based method [11], and proposed method, respectively. The proposed method exactly detects the motion region only, so HDR imaging can be effectively applied to region without motion. In Figure 4(d), the proposed method gives the best performance in view of contrast. Figures 4(a)-4(c) show the color artifact in sky region, which is so-called color shift caused by tone mapping [1], where color distortion can easily occur when the radiance map of luminance is tone-mapped back to fit the range of devices. However, this artifact does not occur in the proposed method because exposure fusion used in the proposed method fuses directly LDRIs.
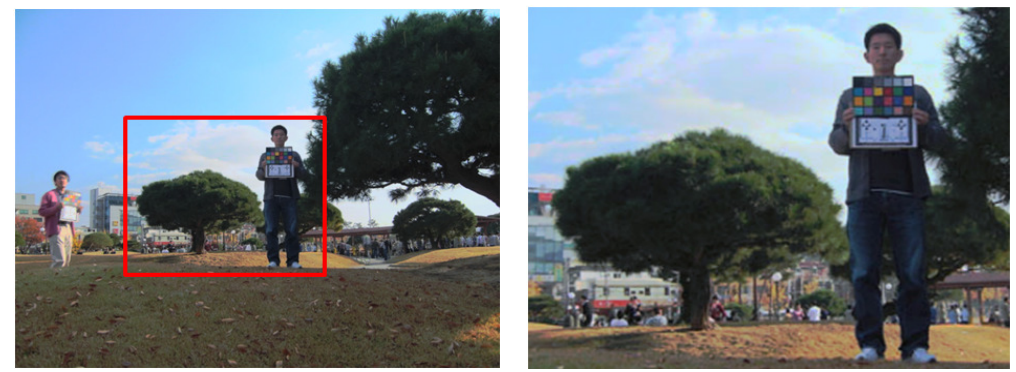

(a) 

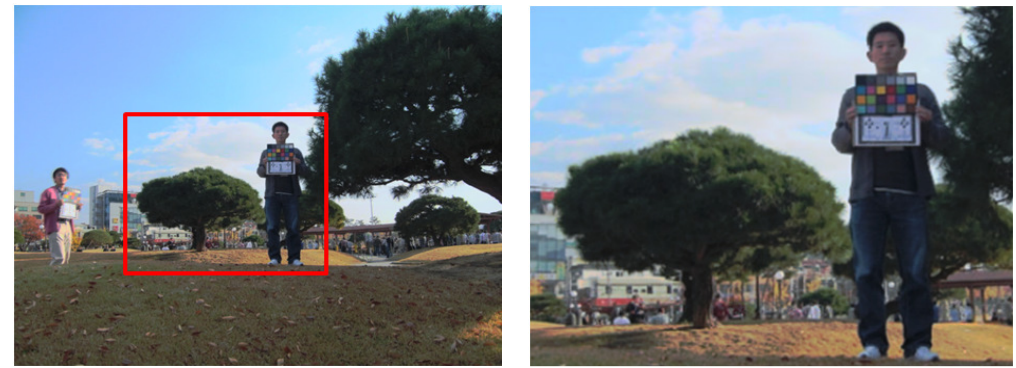

(b)
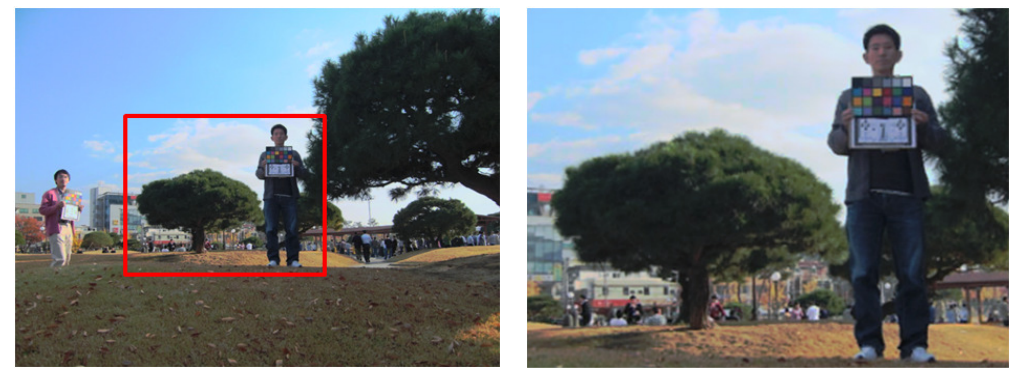

(c)
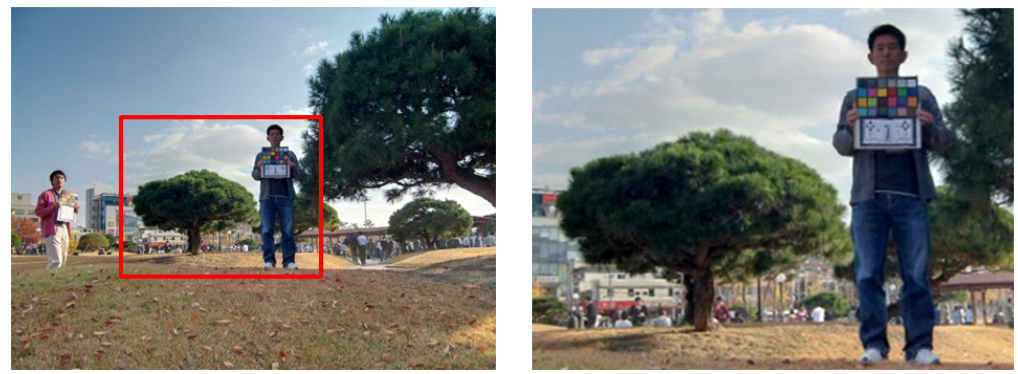

(d)

Figure 4. Comparison of simulation results in view of HDR $(728 \times 546$, Field). (a) variance based method [2], (b) Jacobs et al.'s method [3], (c) adaptive multi-level threshold based method [11], (d) proposed method.

\subsection{Ghost Artifact Removal}

For performance comparison of ghost artifact removal in HDR imaging, three existing methods are used: variance based method [2], Jacobs et al.'s method [3], and tensor voting method [28]. For tone mapping method of variance based method [2] and Jacobs et al.'s method [3], Reinhard et al.'s method [13] is used. These methods $[2,3]$ use the weighted variance and local entropy of pixel values between multiple-exposure LDRIs, respectively. Tensor voting method [28] estimates global and local replacement functions and votes for an optimal replacement function. In our experiments of the proposed method, we set $N=8$ and $T_{\text {rank }}=24$ and a LDRI with middleexposure is selected as reference image for ghost artifact removal. Therefore, the proposed method replaces detected motion region with the region in the reference image.

The performances of ghost artifact removal in region where local motion occurs are compared in Figure 5, where these figures are the cropped $(251 \times 205)$ and enlarged from Figure 1. Figures 5(a) and 5(b) show the result of the variance based method [2] and Jacobs et al.'s method [3], respectively. Note that the result of the proposed method in Figure 5(d) is better than those of the two methods. These existing methods are not robust to motion of an object if the object and background have similar structure. A result by tensor voting method [28] is shown in Figure 5(c), 
where the motion region is replaced by that of under-exposure image, because in this method under-exposure image is used as the reference image in which intensities of under-exposure image are globally and locally aligned to intensities of middle- and over-exposure images. In Figure 5(c), the ghost artifacts remain after HDR imaging. Then, in views of contrast and detailpreservation, the proposed method gives better result than the other methods. In Figure 5(d) by the proposed method, edges, corners, and details are shown sharply. The gain control in the proposed method leads to high contrast and fine details of result HDRI.

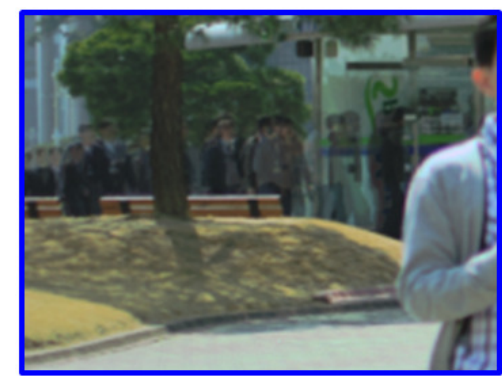

(a)

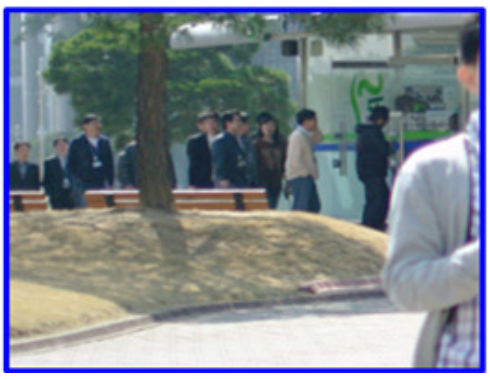

(c)

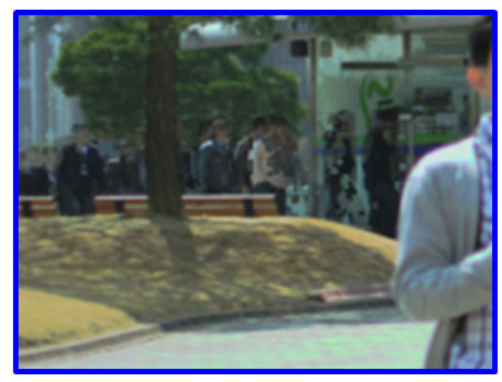

(b)

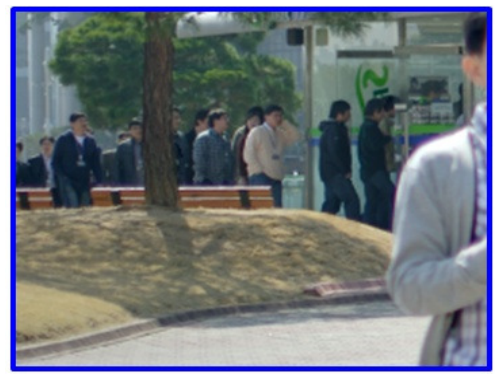

(d)

Figure 5. Comparison of simulation results in view of ghost artifact removal $(251 \times 205$, Bench). (a) variance based method [2], (b) Jacobs et al.'s method [3], (c) tensor voting method [28], (d) proposed method.

\subsection{Noise Removal}

The three existing methods used for comparison in view of noise removal are Debevec and Malik's method [1], Min et al.'s method [11], and Tico et al.'s method [14].

Figure 6(a) shows the noisy LDRI set (Window, 616×462) consisting of three images with different exposures. Figure 6(b) shows the cropped and enlarged region of red boxes in Figure 6(a). We can observe that dark regions in LDRIs with under-exposures are affected by noise.
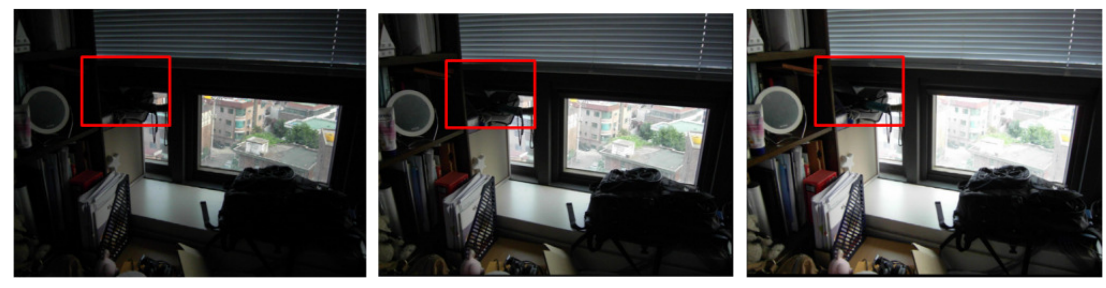

(a) 

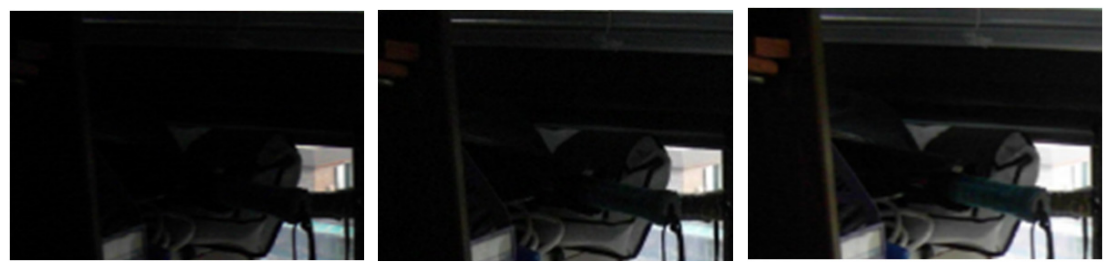

(b)

Figure 6. Noisy LDRIs $(616 \times 462$, Window). (a) input LDRIs, (b) cropped and enlarged regions of LDRIs.

Figure 7 shows the HDR imaging results with noise removal using LDRIs in Figure 6(a). Figures 7(a)-(d) show HDR imaging results by Debevec and Malik's method [1], Min et al.'s method [11], Tico et al.'s method [14], and the proposed method, respectively, where right images are the cropped and enlarged regions from red boxes in left images. Note that Debevec and Malik's method and Min et al.'s method use HDR radiance map generation and tone mapping, whereas Tico et al.'s method and the proposed method use image fusion method. Debevec and Malik used simple weighted average functions for removing noise, however this function is not effective in low light condition as shown in Figure 7(b). Min et al. used the information of spatially and temporally neighboring pixels for noise removal. Although their method gives better results than Debevec and Malik's method, noise still remains as shown in Figure 7(b). Figure 7(c) shows the result of Tico et al.'s method, where noise variance is estimated at each pixel and small weight is assigned to the pixels with large noise variance, however the performance of noise removal is lower than the proposed method in Figure 7(d). In Figure 7(d), the proposed method effectively removes noise as well as preserves edge and color of LDRIs.
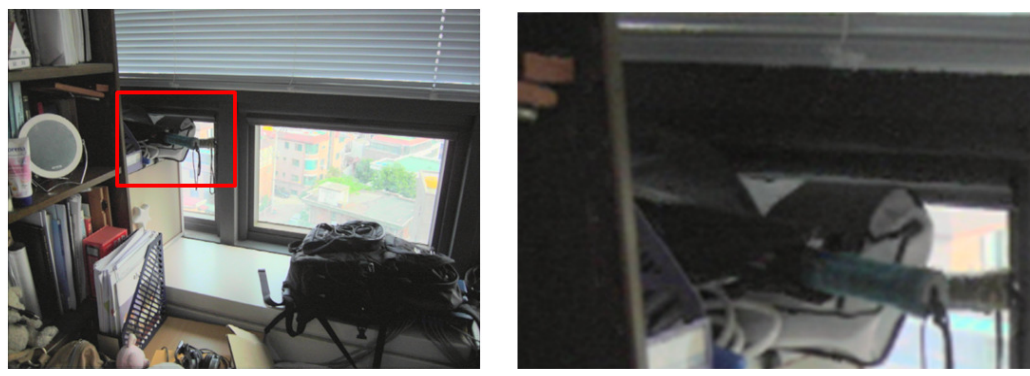

(a)
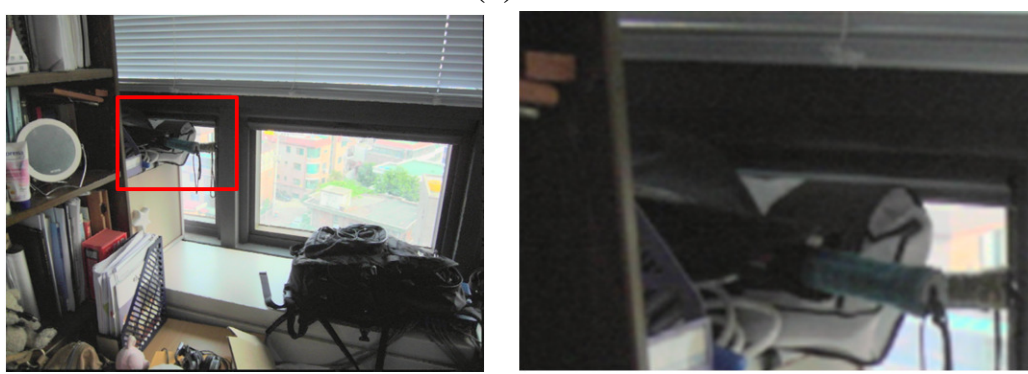

(b) 
International Journal of Computer Graphics \& Animation (IJCGA) Vol.4, No.4, October 2014
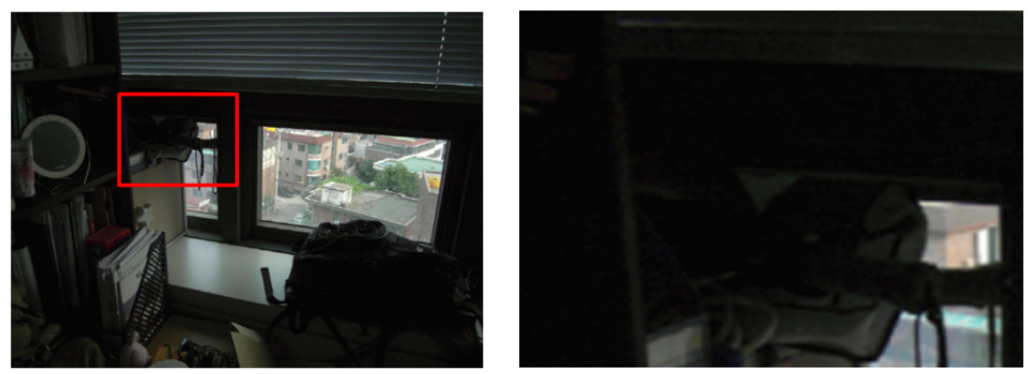

(c)
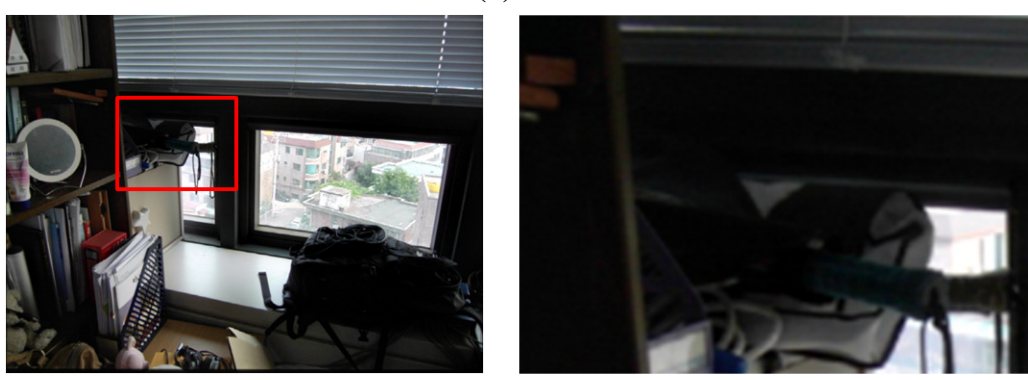

(d)

Figure 7. Comparison of simulation results in view of noise removal $(616 \times 462)$. (a) Debevec and Malik's method [1], (b) Min et al.'s method [11], (c) Tico et al.'s method [14], (d) proposed method.

\subsection{Computation Time}

Table 1 shows the computation times of each process of the proposed method. The proposed motion map generation is useful because of simple implementation and low complexity. It makes the proposed method more practical for today's digital cameras with mega pixel images. However, the processes for denoising and gain control take more time than other processes. Therefore, if the processes of denoising and gain control are adaptively used according to the amount of noise and illumination level, high-quality HDRI can be obtained with less computation.

Table 1. Computation Times of Each Process of the Proposed Method $(563 \times 373 \times 3)$.

\begin{tabular}{cc}
\hline \hline Process & Time $(\mathbf{s e c})$ \\
\hline Decomposition & 2.6573 \\
Motion map generation & 0.4359 \\
Exposure fusion & 6.0985 \\
Denoising & 7.1858 \\
Gain control & 1.2826 \\
Reconstruction & 0.7087 \\
\hline Total & 18.3645 \\
\hline
\end{tabular}

Table 2 shows comparison of the computation time of ghost removal process for three existing and proposed ghost removal methods. We implemented the algorithms in Matlab on an Intel Core i5 $(2.67 \mathrm{GHz}, 4 \mathrm{~GB}$ RAM) and used image set (Field, $728 \times 546 \times 3)$. For ghost removal, the proposed method takes more time than variance based method [2] and multi-level threshold based method [7], however gives better results than three existing methods. 
International Journal of Computer Graphics \& Animation (IJCGA) Vol.4, No.4, October 2014

Table 2. Comparison of the Computation Time of Ghost Removal Process $(728 \times 546 \times 3$, Field $)$.

\begin{tabular}{cc}
\hline \hline Method & Time (sec) \\
\hline Variance based method [2] & 0.7594 \\
Jacobs et al.'s method [3] & 3.2651 \\
Multi-level threshold based method [7] & 0.8257 \\
Proposed method & 1.5170 \\
\hline
\end{tabular}

\section{Conclusions}

We propose a ghost and noise removal method in exposure fusion using subband architecture. Exposure fusion is constructed in the subband architecture and the proposed histogram based motion maps are used for ghost artifact removal caused by local motion, where the motion maps are combined with the weight maps of exposure fusion. Fused subband images are denoised by multi-resolution bilateral filtering and soft threshlolding, and then details of the subband images are enhanced through the gain control. Finally, detail-preserved subband images are reconstructed to a single HDRI. Experimental results show that the proposed method is effective to detect only motion region and to remove noise, while giving good results for HDR imaging. Future research will focus on more practical implementation of the proposed method. In real captured LDRI sets, over-exposure LDRI is affected by blur. Thus, future research will focus on reducing the effect of blur image degradation in generating a single high-quality HDRI.

\section{ACKNOWLEDGEMENTS}

This work was supported in part by Digital Imaging Business, Samsung Electronics, Co. Ltd.

\section{REFERENCES}

[1] P. Debevec and J. Malik, "Recovering high dynamic range radiance maps from photographs", Proc. SIGGRAPH, Los Angeles, CA, Aug. 1997, pp. 369-378.

[2] E. Reinhard, G. Ward, P. Debevec, and S. Pattanaik, High Dynamic Range Imaging: Acquisition, Display, and Image Based Lighting, Morgan Kaufmann, San Francisco, CA, 2006.

[3] K. Jacobs, C. Loscos, and G. Ward, "Automatic high dynamic range image generation for dynamic scenes", IEEE Computer Graphics and Applications, Vol. 28, No. 2, Mar. 2008, pp. 84-93.

[4] A. A. Goshtasby, "Fusion of multi-exposure images", Image and Vision Computing, Vol. 23, No. 6, June 2005, pp. 611-618.

[5] T. Martens, J. Kautz, and F. V. Reeth, "Exposure fusion: A simple and practical alternative to high dynamic range photography”, Computer Graphics Forum, Vol. 28, No. 1, Mar. 2009, pp. 161-171.

[6] G. Ward, "Fast, robust image registration for compositing high dynamic range photographs for handheld exposures", Journal of Graphics Tools, Vol. 8, No. 2, Jan. 2003, pp. 17-30.

[7] T.-H. Min, R.-H. Park, and S. Chang, "Histogram based ghost removal in high dynamic range images", Proc. IEEE Int. Conf. Multimedia and Expo, New York, June/July 2009, pp. 530-533.

[8] W. Zhang and W.-K. Cham, "Gradient-directed composition of multi-exposure images," Proc. IEEE Conf. Computer Vision and Pattern Recognition, San Francisco, CA, June 2010, pp. 530-536.

[9] S. B. Kang, M. Uyttendaele, S. Winder, and R. Szeliski, "High dynamic range video", ACM Trans. Graphics, Vol. 22, No. 3, July 2003, pp. 319-325.

[10] A. O. Akyuz and E. Reinhard, "Noise reduction in high dynamic range imaging", Journal of Visual Communication and Image Representation, Vol. 18, No. 5, Oct. 2007, pp. 366-376. 
International Journal of Computer Graphics \& Animation (IJCGA) Vol.4, No.4, October 2014

[11] T.-H. Min, R.-H. Park, and S. Chang, "Noise reduction in high dynamic range images", Signal, Image, and Video Processing, Vol. 5, No. 3, Sept. 2011, pp. 315-328.

[12] A. A. Bell, C. Seiler, J. N. Kaftan, and T. Aach, "Noise in high dynamic range imaging", Proc. Int. Conf. Image Processing, San Diego, CA, Oct. 2008, pp. 561-564.

[13] E. Reinhard, M. Stark, P. Shirley, and J. Ferwerda, "Photographic tone reproduction for digital images", ACM Trans. Graphics, Vol. 21, No. 3, July 2002, pp. 267-273.

[14] M. Tico, N. Gelfand, and K. Pulli, "Motion-blur-free exposure fusion", in Proc. Int. Conf. Image Processing, Hong Kong, China, Sept. 2010, pp. 3321-3324.

[15] S. Raman and S. Chaudhuri, "Bilateral filter based compositing for variable exposure photography", Proc. Eurographics Conf., Munich, Germany, Mar. 2009, pp. 1-4.

[16] B.-D. Choi, S.-W. Jung, and S.-J. Ko, "Motion-blur-free camera system splitting exposure time", IEEE Trans. Consumer Electronics, Vol. 54, No. 3, Aug. 2008, pp. 981-986.

[17] J. Jia, J. Sun, C.-K. Tang, and H.-Y. Shum, "Bayesian correction of image intensity with spatial consideration", Proc. 8th European Conf. Computer Vision, Lecture Notes in Computer Science, Vol. 3023, Prague, Czech Republic, May 2004, pp. 342-354.

[18] D.-K. Lee, R.-H. Park, and S. Chang, "Improved histogram based ghost removal in exposure fusion for dynamic range images", Proc. 15th IEEE Int. Symp. Consumer Electronics, Singapore, June 2011, pp. 586-591.

[19] M. Zhang and B. Gunturk, "Multiresolution bilateral filtering for image denoising", IEEE Trans. Image Processing, Vol. 17, No. 12, Dec. 2008, pp. 2324-2333.

[20] J. Shen, Y. Zhao, and Y. He, "Detail-preserving exposure fusion using subband architecture", The Visual Computer, Vol. 28, No. 5, May 2012, pp. 463-473.

[21] S. Lee, "Compressed image reproduction based on block decomposition”, IET Image Processing, Vol. 3, No. 4, Aug. 2009, pp. 188-199.

[22] X. Li, F. Li, L. Zhuo, and D. D. Feng, "Layers-based exposure fusion algorithm”, IET Image Processing, Vol. 7, No. 7, Oct. 2013, pp. 701-711.

[23] M. Bertalmio and S. Levine, "Variational Approach for the Fusion of Exposure Bracketed Pairs", IEEE Trans. Image Processing, Vol. 22, No. 2, Feb. 2013, pp. 712-723.

[24] Y. Li, L. Sharan, and E. H. Adelson, "Compressing and companding high dynamic range images with subband architectures”, ACM Trans. Graphics, Vol. 24, No. 3, July 2005, pp. 836-844.

[25] S. Baker and I. Matthews, "Lucas-Kanade 20 years on: A unifying framework", Int. Journal of Computer Vision, Vol. 56, No. 3, Feb. 2004, pp. 221-255.

[26] Y. Keller and A. Averbuch, "Fast gradient methods based on global motion estimation for video compression”, IEEE Trans. Circuits Syst. Video Technol., Vol. 13, No. 4, Apr. 2003, pp. 300-309.

[27] E. A. Khan, A. O. Akyuz, and E. Reinhard, "Robust generation of high dynamic range images", Proc. Int. Conf. Image Processing, Atlanta, GA, Oct. 2006, pp. 2005-2008.

[28] J. Jia and C. K. Tang, "Tensor voting for image correction by global and local intensity alignment", IEEE Trans. Pattern Analysis and Machine Intelligence, Vol. 27, No. 1, Jan. 2005, pp. 36-50.

[29] P. J. Burt and E. H. Adelson, "The Laplacian pyramid as a compact image code", IEEE Trans. Communications, Vol. 31, No. 4, Apr. 1983, pp. 532-540.

[30] R. C. Gonzalez and R. E. Woods, Digital Image Processing. 3rd ed., Upper Saddle River, NJ: Pearson Education Inc., 2010.

[31] D. Donoho and I. Johnstone, "Ideal spatial adaptation by wavelet shrinkage", Biometrika, Vol. 81, No. 3, Aug. 1994, pp. 425-455.

[32] C. Tomasi and R. Manduchi, "Bilateral filtering for gray and color images", Proc. Int. Conf. Computer Vision, Bombay, India, Jan. 1998, pp. 839-846.

[33] S. G. Chang, B. Yu, and M. Vetterli, "Adaptive wavelet thresholding for image denoising and compression”, IEEE Trans. Image Processing, Vol. 9, No. 9, Sept. 2000, pp. 1532-1546.

[34] Y. Yoo, W. Choe, J. Kwon, S. Park, S. Lee, and C.-Y. Kim, "Low-light imaging method with visibleband and wide band image pair", Proc. Int. Conf. Image Processing, Cairo, Egypt, Nov. 2009, pp. 2273-2276. 
International Journal of Computer Graphics \& Animation (IJCGA) Vol.4, No.4, October 2014

\section{Authors}

Dong-Kyu Lee received the B.S. and M.S. degrees in electronic engineering from Sogang University in 2010 and 2012, respectively. His current research interests are image processing and image enhancement.

Rae-Hong Park received the B.S. and M.S. degrees in electronics engineering from Seoul National University, Seoul, Korea, in 1976 and 1979, respectively, and the M.S. and Ph.D. degrees in electrical engineering from Stanford University, Stanford, CA, in 1981 and 1984, respectively. In 1984, he joined the faculty of the Department of Electronic Engineering, School of Engineering, Sogang University, Seoul, Korea, where he is currently a Professor. In 1990, he spent his sabbatical year as a Visiting Associate Professor with the Computer Vision Laboratory, Center for Automation Research, University of Maryland at College Park. In 2001 and 2004, he spent sabbatical semesters at Digital Media Research and Development Center (DTV image/video enhancement), Samsung Electronics Co., Ltd., Suwon, Korea. In 2012, he spent a sabbatical year in Digital Imaging Business (R\&D Team) and Visual Display Business (R\&D Office), Samsung Electronics Co., Ltd., Suwon, Korea. His current research interests are video communication, computer vision, and pattern recognition. He served as Editor for the Korea Institute of Telematics and Electronics (KITE) Journal of Electronics Engineering from 1995 to 1996. Dr. Park was the recipient of a 1990 Post-Doctoral Fellowship presented by the Korea Science and Engineering Foundation (KOSEF), the 1987 Academic Award presented by the KITE, the 2000 Haedong Paper Award presented by the Institute of Electronics Engineers of Korea (IEEK), the 1997 First Sogang Academic Award, and the 1999 Professor Achievement Excellence Award presented by Sogang University. He is a co-recipient of the Best Student Paper Award of the IEEE Int. Symp. Multimedia (ISM 2006) and IEEE Int. Symp. Consumer Electronics (ISCE 2011).

SoonKeun Chang received his B.S. degree in astronomy and space science from KyungHee University, Korea, in 2000 and M.S. degree in control engineering from Kanazawa University in 2003. He received a Ph.D. degree in control engineering from Tokyo Institute of Technology (TITech) in 2007. Now he works at Samsung Electronics Co., Ltd., Korea. His main research interests include computer vision and image processing. 